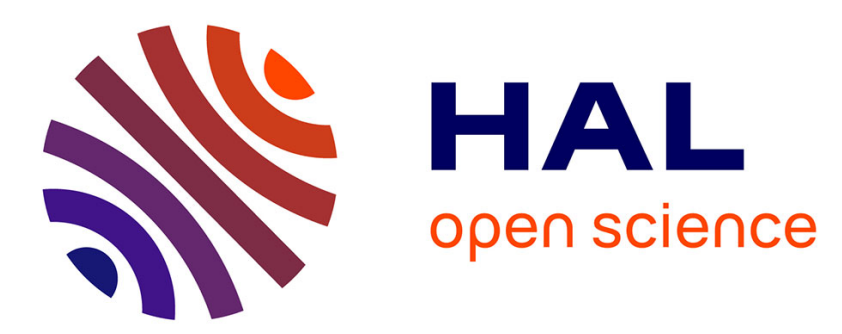

\title{
Spatially-variant area openings for reference-driven adaptive contour preserving filtering
}

\author{
Gianni Franchi, Jesus Angulo
}

\section{To cite this version:}

Gianni Franchi, Jesus Angulo. Spatially-variant area openings for reference-driven adaptive contour preserving filtering. 2014 22nd International Conference on Pattern Recognition (ICPR), Aug 2014, Stockholm, Sweden. 10.1109/ICPR.2014.189 . hal-00926681

\section{HAL Id: hal-00926681 \\ https://hal.science/hal-00926681}

Submitted on 10 Jan 2014

HAL is a multi-disciplinary open access archive for the deposit and dissemination of scientific research documents, whether they are published or not. The documents may come from teaching and research institutions in France or abroad, or from public or private research centers.
L'archive ouverte pluridisciplinaire HAL, est destinée au dépôt et à la diffusion de documents scientifiques de niveau recherche, publiés ou non, émanant des établissements d'enseignement et de recherche français ou étrangers, des laboratoires publics ou privés. 


\section{Spatially-variant area openings for reference-driven adaptive contour preserving filtering}

\author{
Gianni Franchi \\ CMM-Centre de Morphologie Mathématique \\ MINES ParisTech, Paris, France \\ Email: gianni.franchi@mines-paristech.fr
}

\author{
Jesús Angulo \\ CMM-Centre de Morphologie Mathématique \\ MINES ParisTech, Paris, France \\ Email: jesus.angulo@mines-paristech.fr
}

\begin{abstract}
Classical adaptive mathematical morphology is based on operators which locally adapt the structuring elements to the image properties. Connected morphological operators act on the level of the flat zones of an image, such that only flat zones are filtered out, and hence the object edges are preserved. Area opening (resp. area closing) is one of the most useful connected operators, which filters out the bright (resp. dark) regions. It intrinsically involves the adaptation of the shape of the structuring element parameterized by its area. In this paper, we introduce the notion of reference-driven adaptive area opening according to two spatially-variant paradigms. First, the parameter of area is locally adapted by the reference image. This approach is applied to processing intensity+depth images where the depth image is used to adapt the scale-size processing. Second, a self-dual area opening, where the reference image determines if the area filter is an opening or a closing with respect to the relationship between the image and the reference. Its natural application domain are the video sequences.
\end{abstract}

\section{INTRODUCTION}

Area opening (resp. area closing) is a morphological filter that removes from an image the bright (resp. dark) connected components having a surface area smaller than the parameter $\lambda$ [13]. Area openings on gray-level images can be implemented from an upper level set decomposition as well as using more efficient algorithms based on max-tree [8] or component tree [4] representations. For a recent overview of applications of area openings, see [6]. It is also possible to formulate area-based operators which simultaneously filter out bright and dark connected components and consequently being self-dual [10]. The same effect can be obtained by working a tree representation of the image called Fast Level Lines Transform [3].

The main interest of area opening resides in the fact that they can be seen as morphological openings with a structuring element which locally adapts its shape to the image structures and consequently the contours of the objects are not deformed. This is the common property of connected operators [7]. Classical adaptive mathematical morphology is based on operators which locally adapts the structuring elements to the image properties, see state-of-the-art of adaptive morphology in recent papers [11] [2].

Hence, area opening can be seen as an adaptive operator. However area opening takes just the information from one image. In this article, we formulate two variant of area opening that can deal with two images, one image where we would apply the area filter and the other one, called the reference, introduces an external adaptivity of the operator. These formulations can be considered as reference-driven adaptive area opening according to two different spatially-variant paradigms. First, the parameter of area is locally adapted by the reference image. This approach is applied to processing intensity+depth images where the depth image is used to adapt the size scale processing. Second, a self-dual area opening, where the reference image determines if the area filter is an opening or a closing with respect to the relationship between the image and the reference. Its natural application domain are the video sequences. The present adaptive area openings are defined according to the framework of grain filters by Boolean predicates introduced in [15].

Note that our self-dual area opening according to a reference is related to the levelings [5], but in our case we deal with an area criterion. In a recent work [9], it has been proposed a framework for adaptive connected operators based on a reconstruction from a marker propagated over increasing quasi-flat zones according to an attribute, then a selection on a hierarchical representation of the attribute value leading to the strongest change. This idea is related to the notion of ultimate opening [1], which in fact do not produce a transform having the properties of an algebraic opening. We notice that in our case, the proposed adaptive area openings have the needed algebraic properties. In addition, the adaptability of the area value is parameterized by an external reference and the corresponding size-adaptive opening can be straightforward formulated in an upper level set decomposition framework.

\section{BACKGROUND ON AREA OPENINGS}

Let $E$ be a subset of the discrete space $\mathbb{Z}^{2}$, which represents the support space of a $2 \mathrm{D}$ image and $\mathcal{T} \subseteq \mathbb{R}$ be the set of intensity pixels values. Hence, it is assumed that the value at a pixel position $x \in E$ is represented by a scalar grey-level intensity $s \in \mathcal{T}$ by means of the function $f: E \rightarrow \mathcal{T}$.

\section{A. Algebraic openings in mathematical morphology}

Let $f \in \mathcal{F}(E, \mathcal{T})$ be a grey-scale image. Morphological opening of $f$ according to structuring element $B$ is defined as $\gamma_{B}(f)=\delta_{B}\left(\varepsilon_{B}(f)\right)$, where $\varepsilon_{B}(f)$ and $\delta_{B}(f)$ are respectively the erosion and the dilation of $f$ by the flat structuring element $B$ [17]. More generally, an algebraic opening $\gamma(f)$ is any operator on $f$ following these three properties [17]: (a) increasing, i.e., if $f \leq g$ then $\gamma(f) \leq \gamma(g)$; (b) idempotent, i.e., $\gamma(\gamma(f))=\gamma(f)$; (c) anti-extensive, i.e., $\gamma(f) \leq f$. 
The latter property means that bright structures are removed from the image. Similarly, an algebraic closing $\varphi$ is any operator $\mathcal{F}(E, \mathcal{T}) \rightarrow \mathcal{F}(E, \mathcal{T})$ being increasing, idempotent and extensive (i.e., acting on dark structures).

\section{B. Upper level sets and area opening for grey-level images}

Area opening is a typical example of algebraic opening. Given a binary image $b$, which can be represented by the set of the finite union of its connected components of value 1, i.e., $\mathcal{C}_{b}^{+}=\cup_{k} C_{k}$ such that $b\left(\left\{C_{k}\right\}\right)=1$, the area opening of size $0<\lambda \in \mathbb{N}$ is defined as follows [13]:

$$
\gamma_{\lambda}(b)=\bigcup_{k}\left\{C_{k} \mid \operatorname{area}\left(C_{k}\right) \geq \lambda\right\}
$$

Therefore, $\gamma_{\lambda}(b)$ is the union of the connected components of $b$ with area greater or equal than $\lambda$. By area is meant the Lebesgue measure in $\mathbb{Z}^{2}$. This operator is equivalent to

$$
\gamma_{\lambda}(b)=\bigvee_{i}\left\{\gamma_{B_{i}}(f) \mid B_{i} \text { is connected and } \operatorname{card}\left(B_{i}\right)=\lambda\right\}
$$

where consequently $B_{i}$ is a structuring element. The proof of this result can be find in [13]. Obviously, computing the area opening according to (2) is an impossible task. But, as a supremum of openings, it gives an interpretation of the area opening as an adaptive operator: at every location, the structuring element adapts its shape to image structure.

Area openings are naturally extended to grey-scale images [13]. Since we are going to work on the connected components of grey-scale image $f \in \mathcal{F}(E, \mathcal{T})$, it is common to decompose it into its upper level sets, where the upper level set for a given threshold $s \in \mathcal{T}$ and a given image $f$ is the binary image defined as:

$$
X_{s}(f)(x)=\left\{\begin{array}{l}
1 \text { if } f(x) \geq s \\
0 \text { if } f(x)<s
\end{array}\right.
$$

Using now the family of upper level set, we can easily obtain the original image as [12]:

$$
f=\sum_{s \in \mathcal{T}} X_{s}(f)
$$

Our interest in upper level sets can be explained by the fact that there are many interesting properties linking upper level set with flat morphological operators [12]. Namely, if we have defined a binary area opening $\Gamma_{\lambda}$ with the attribute value $\lambda$, the corresponding grey-scale area opening $\gamma_{\lambda}$ of image $f$ is given by

$$
\gamma_{\lambda}(f)=\sum_{s \in \mathcal{T}} \Gamma_{\lambda}\left(X_{s}(f)\right)
$$

For the sake of pedagogy, we show here how to check that $\gamma_{\lambda}$ is an algebraic opening since a similar mechanism will be used for the proposed adaptive area openings. More precisely, we should prove that $\gamma_{\lambda}$ is increasing, anti-extensive and idempotent.

a) Increasing: Let us consider $f$ and $g$ two grey-scale images such as $f \leq g$. So for all $s \in \mathcal{T}, X_{s}(f) \leq X_{s}(g)$. Since $\Gamma_{\lambda}$ is an opening we have: $\Gamma_{\lambda}\left(X_{s}(f)\right) \leq \Gamma_{\lambda}\left(X_{s}(g)\right)$, thus $\sum_{s \in \mathcal{T}} \Gamma_{\lambda}\left(X_{s}(f)\right) \leq \sum_{s \in \mathcal{T}} \Gamma_{\lambda}\left(X_{s}(g)\right)$. Hence we have $\gamma_{\lambda}\left(X_{s}(f)\right) \leq \gamma_{\lambda}\left(X_{s}(g)\right)$. Hence this operator is increasing. b) Anti-extensive: Let us consider $f$ a grey-scale image. For all $s \in \mathcal{T}, \Gamma_{\lambda}\left(X_{s}(f)\right) \leq X_{s}(f)$ since $\Gamma_{\lambda}$ is an opening. Hence $\sum_{s \in \mathcal{T}} \Gamma_{\lambda}\left(X_{s}(f)\right) \leq \sum_{s \in \mathcal{T}} X_{s}(f)$. So $\gamma_{\lambda}(f) \leq f$. Hence this operator is anti-extensive.

c) Idempotent: Let us consider $f$ a grey-scale image. Since $\gamma_{\lambda}$ is anti-extensive we have:

$$
\gamma_{\lambda}\left(\gamma_{\lambda}(f)\right) \leq \gamma_{\lambda}(f)
$$

Moreover $\gamma_{\lambda}\left(\gamma_{\lambda}(f)\right)=\gamma_{\lambda}\left(\sum_{s_{2} \in \mathcal{T}} \Gamma_{\lambda}\left(X_{s_{2}}(f)\right)\right)$, hence we have $\gamma_{\lambda}\left(\gamma_{\lambda}(f)\right)=\sum_{s_{1} \in \mathcal{T}} \Gamma_{\lambda}\left(X_{s_{1}}\left(\sum_{s_{2} \in \mathcal{T}} \Gamma_{\lambda}\left(X_{s_{2}}(f)\right)\right)\right)$. Since $X_{s_{1}}\left(\sum_{s_{2} \in \mathcal{T}} \Gamma_{\lambda}\left(X_{s_{2}}(f)\right)\right)=\sum_{s_{2} \in \llbracket s_{1} ; N \rrbracket .} \Gamma_{\lambda}\left(X_{s_{2}}(f)\right)$, where $\operatorname{card}(\mathcal{T})=N$. So easily we have $\sum_{s_{2} \in \llbracket s_{1} ; N \rrbracket .} \Gamma_{\lambda}\left(X_{s_{2}}(f)\right) \quad \geq \quad \Gamma_{\lambda}\left(X_{s_{1}}(f)\right)$, hence $\quad \sum_{s_{1} \in \mathcal{T}} \Gamma_{\lambda}\left(\sum_{s_{2} \in \llbracket s_{1} ; N \rrbracket} \Gamma_{\lambda}\left(X_{s_{2}}(f)\right)\right) \quad \geq$ $\sum_{s_{1} \in \mathcal{T}} \Gamma_{\lambda}\left(\Gamma_{\lambda}\left(X_{s_{1}}(f)\right)\right)=\sum_{s_{1} \in F} \Gamma_{\lambda}\left(X_{s_{1}}(f)\right)$. So we have

$$
\gamma_{\lambda}\left(\gamma_{\lambda}(f)\right) \geq \gamma_{\lambda}(f)
$$

Hence thanks to (6) and (7) this operator is idempotent and we now conclude that $\gamma_{\lambda}$ is an algebraic opening.

\section{SizE AdAptive AREA OPENING}

\section{A. Definition}

Let us consider two grey-level images $f$ and $r$. It can be interested to develop an area opening on $f$ which will depend locally on the information driven by $r$. In order to have an operator that do not cut objects, we choose to work on connected components. Thus we start by decomposing $f$ into its upper level sets $\left\{X_{s}(f)\right\}_{s \in \mathcal{T}}$. We are now going first to write our opening for binary images, then just by a similar demonstration to the previous one, the addition of this binary opening on all the upper level set would still be an opening. That is how we would create our size adaptive area opening on grey-scale images.

For now let us consider $f$ as a binary image, and the reference $r$ as a grey-scale image. Let us decompose $f \in$ $\mathcal{F}(E,\{1,0\})$, in a set of connected objects called $\mathcal{C}_{f}^{+}$, where there are all the connected components with positive pixels, and in another set $\mathcal{C}_{f}^{0}$, where there are all the connected components with zero pixels, such as $f=\mathcal{C}_{f}^{+} \cup \mathcal{C}_{f}^{0}$. We introduce a Boolean function $v: \mathcal{C}_{f}^{+} \rightarrow\{1,0\}$ that measures the area of positive connected component $C_{k} \in \mathcal{C}_{f}^{+}$and checks if it is greater than $\lambda\left(r, C_{k}\right)$. Hence, for all $C_{k} \in \mathcal{C}_{f}^{+}$we have:

$$
v\left(C_{k}\right)=\left\{\begin{array}{l}
1 \text { if area }\left(C_{k}\right) \geq \lambda\left(r, C_{k}\right) \\
0 \text { otherwise }
\end{array}\right.
$$

For our application, we need to define also another Boolean function $u: \mathcal{C}_{f}^{+} \rightarrow\{1,0\}$ such as for all $C_{k} \in \mathcal{C}_{f}^{+}$we have:

$$
u\left(C_{k}\right)=\left\{\begin{array}{l}
1 \text { if } \operatorname{var}\left(r\left(C_{k}\right)\right) \leq \alpha \\
0 \text { otherwise }
\end{array}\right.
$$

where $\operatorname{var}\left(r\left(C_{k}\right)\right)$ represents the variance of image $r$ inside the connected component $C_{k}$ and $\alpha$ is a threshold. This choice of $u$ was motivated by the fact that the adaptation in size for a connected component in $f$ makes sense only if the variation in $C_{k}$ of the reference image is limited. Other alternative functions $u$ can be considered for the same purpose. 
Therefore, we can now define the area opening on the binary image $f$ driven by the reference $r$ as:

$\widetilde{\gamma}_{\lambda, r}(f)=\bigcup_{k}\left\{C_{k} \mid\left(C_{k} \in \mathcal{C}_{f}^{+}\right) \&\left(u\left(C_{k}\right)=1\right) \&\left(v\left(C_{k}\right)=1\right)\right\}$.

Using (5), we formulate size adaptive area opening $\widetilde{\gamma_{\lambda, r}}(f)$ on a grey-scale image $f \in \mathcal{F}(E, \mathcal{T})$ as the sum of the previous binary opening applied in all its upper level sets:

$$
\widetilde{\gamma_{\lambda, r}}(f)(x)=\sum_{s \in \mathcal{T}} \widetilde{\gamma_{\lambda, r}}\left(X_{s}(f)\right)(x) \text {. }
$$

\section{B. Proof of the opening}

Let us demonstrate that operator (10) is an algebraic opening, and consequently that (11) is also an opening.

d) Anti-extensive: Image $f$ is binary, so is its result $\widetilde{\gamma}_{\lambda, r}$. Hence we have $\widetilde{\gamma}_{\lambda, r}(f)(C)=\mathcal{C}_{\widetilde{\gamma}_{\lambda, r}(f)}^{+} \cup \mathcal{C}_{\widetilde{\gamma}_{\lambda, r}(f)}^{0}$.

Let us consider $\widetilde{\gamma}_{\lambda, r}(f)(C) \in \mathcal{C}_{\widetilde{\gamma}_{\lambda, r}(f)}^{+}$, in this case thanks to the definition of the opening we have $C \in \mathcal{C}_{f}^{+}$so $\mathcal{C}_{\widetilde{\gamma}_{\lambda, r}(f)}^{+} \subseteq$ $\mathcal{C}_{f}^{+}$. Then it means that $\widetilde{\gamma}_{\lambda, r}(f) \leq f$.

e) Increasing: Let us consider two binary images $f$ and $g$ such as $f \leq g$, so $\mathcal{C}_{f}^{+} \subseteq \mathcal{C}_{g}^{+}$. Let us assume that $\widetilde{\gamma}_{\lambda, r}(f)(C) \in \mathcal{C}_{\widetilde{\gamma}_{\lambda, r}(f)}^{+}$. In this case we have $C \in \mathcal{C}_{f}^{+}$, $v(C)=1$ and $u(C)=1$. Easily, thanks to the fact that $\mathcal{C}_{f}^{+} \subseteq \mathcal{C}_{g}^{+}$, we have $\widetilde{\gamma}_{\lambda, r}(f)(C) \in \mathcal{C}_{\widetilde{\gamma}_{\lambda, r}(g)}^{+}$. That means that $\mathcal{C}_{\widetilde{\gamma}_{\lambda, r}(f)}^{+} \subseteq \mathcal{C}_{\widetilde{\gamma}_{\lambda, r}(g)}^{+}$, hence $\widetilde{\gamma}_{\lambda, r}(f)(C) \leq \widetilde{\gamma}_{\lambda, r}(f)(C)$.

f) Idempotent: Let us consider a binary image $f$. Since $\widetilde{\gamma}_{\lambda}$ is anti-extensive we have:

$$
\widetilde{\gamma}_{\lambda, r}\left(\widetilde{\gamma}_{\lambda, r}(f)\right) \leq \widetilde{\gamma}_{\lambda, r}(f)
$$

Let us consider $\widetilde{\gamma}_{\lambda, r}\left(\widetilde{\gamma}_{\lambda, r}(f)\right)(C) \in \mathcal{C}_{\widetilde{\gamma}_{\lambda, r}}\left(\widetilde{\gamma}_{\lambda, r}(f)\right)$, since the definition of the opening $\widetilde{\gamma}_{\lambda, r}(f)(C) \in \mathcal{C}_{\widetilde{\gamma}_{\lambda, r}^{+}(f)}$. Thanks to the definition of the opening $\widetilde{\gamma}_{\lambda, r}\left(\widetilde{\gamma}_{\lambda, r}(f)\right)(C) \in \mathcal{C}_{\widetilde{\gamma}_{\lambda, r}(f)}^{+}$, thus $\mathcal{C}_{\widetilde{\gamma}_{\lambda, r}\left(\widetilde{\gamma}_{\lambda, r}(f)\right)}^{+} \subseteq \mathcal{C}_{\widetilde{\gamma}_{\lambda, r}(f)}^{+}$and hence

$$
\widetilde{\gamma}_{\lambda, r}\left(\widetilde{\gamma}_{\lambda, r}(f)\right) \geq \widetilde{\gamma}_{\lambda, r}(f)
$$

Then we can conclude that $\widetilde{\gamma}_{\lambda, r}$ is idempotent and finally, together with d) and e), that our operator is an opening.

\section{Application to adaptively filter out $R G B-D$ images}

In this section we consider the application of size adaptive area openings to processing intensity (or color) images using the range or depth image. Therefore, let us imagine we have two images from the same scene, one image would provide visual information and the other one would provide the $3 \mathrm{D}$ depth information. This is typically the case of the images produced by the well-known Kinect camera.

To illustrate its performance, we use the example depicted in Figure 2. If one use the classical area opening on this example, as given in images (c) and (e), the opening would act independently of the distance of the real object from the camera.

However, projection camera model based on geometrical optics implies that further an object is from the camera smaller it is, and then by applying an area opening of attribute $\lambda$ we will remove objects whose size in pixel is smaller than this attribute. But we would not take into account its real size in comparison with the same object closer to the camera. That is why we implemented a attribute $\lambda(r, C)$ that depends on the depth of the image. By this approach, we can process objects on the image invariantly to their distance to the camera, and without an explicit 3D reconstruction of the scene. For our application, we have use a parametrization of the surface area as the function $\lambda(r, C)$ such as for all $C \in \mathcal{C}_{f}^{+}$we have:

$$
\lambda(r, C)=\frac{3}{4} \cdot \lambda \cdot e^{(\min (r)-r(C))}+\frac{\lambda}{4}
$$

This function is quite simple but as one could see in Figure 1 , this new attribute would not be constant, and would vary according its depth information. Here we simply assume that the radius of the object is proportional to the distance and that the area grows with respect to the square of radius. A possibility of improvement involves to use a more appropriate model for the camera.

If we observe the size adaptive area openings, images (d) and (f) in Figure 2, we note that as expected the bright structures are simplified, without deforming the contours of the objects, but the effect of the simplification is adapted to the distance such as large objects far from the camera are not removed.

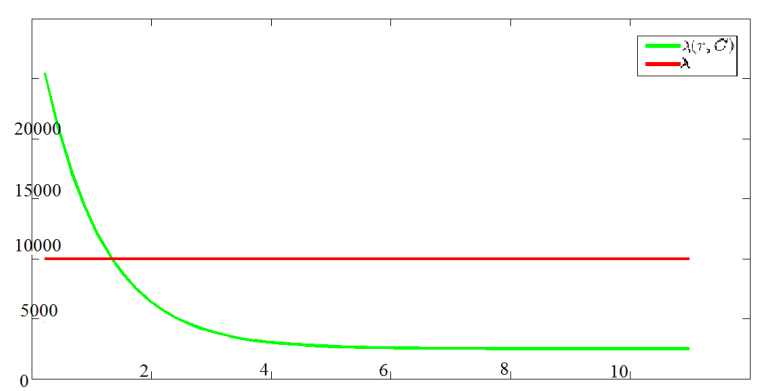

Fig. 1. Example of attribute function $\lambda(r, C)$, where on the abscissa one has the depth scale, which is between 1 and 10 for a typical Kinect camera scene, and on the ordinate the value of the area.

\section{Self-Dual AdAptive Area Opening}

\section{A. Self-dual morphological operators}

Mathematical morphology was initially developed for binary images, and the first morphological operators were the erosion and the dilation. These transforms are dual between them. It means that applying a dilation on an image $f$ is the same as applying an erosion on the complement of $f$ and then doing the complement of this result, where the complement is a way to inverse the role of the foreground and the background, i.e.

$$
\begin{aligned}
& \varepsilon_{B}(f)(x)=\complement \delta_{B}(\complement f)(x), \\
& \delta_{B}(f)(x)=\complement \varepsilon_{B}(\complement f)(x),
\end{aligned}
$$

where $\complement f$ denotes the complementary of $f$. Hence the effect of a morphological operator depends on what is foreground on the image and on what is background. Moreover their effects on foreground are different than those on background. 


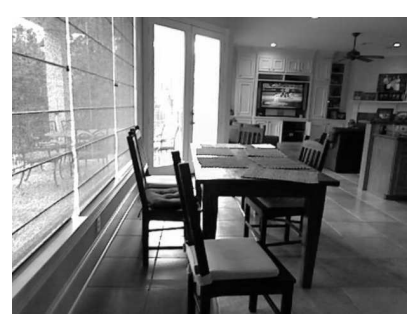

(a) image $f$

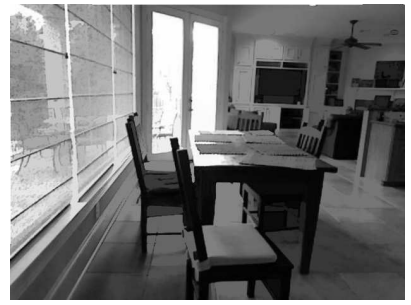

(c) $\gamma_{2500, r}(f)$

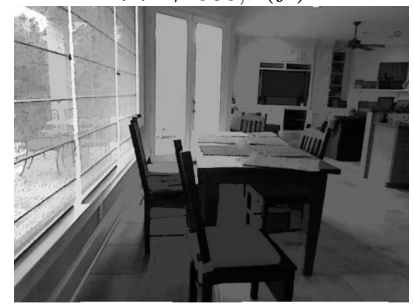

(e) $\gamma_{10000, r}(f)$

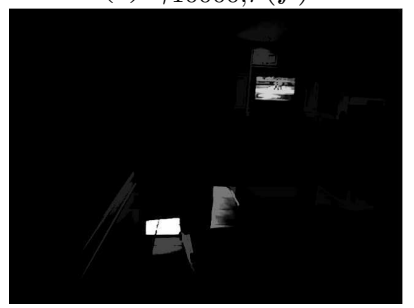

(g) $\gamma_{2500, r}(f)-\widetilde{\gamma}_{2500, r}(f)$

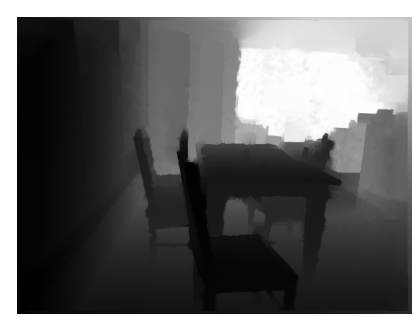

(b) reference $r$

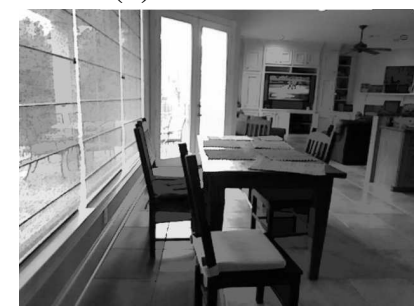

(d) $\widetilde{\gamma}_{2500, r}(f)$

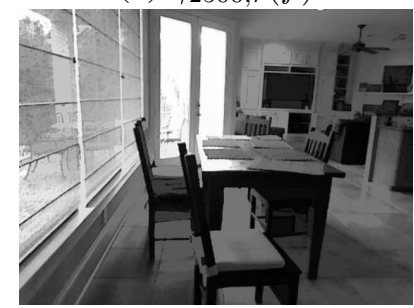

(f) $\widetilde{\gamma}_{10000, r}(f)$

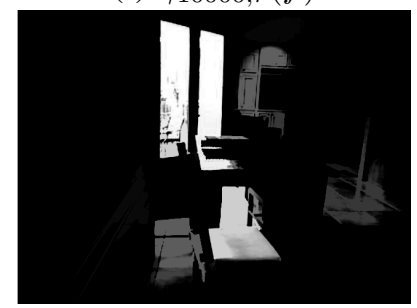

(h) $\gamma_{10000, r}(f)-\widetilde{\gamma}_{10000, r}(f)$
Fig. 2. Examples of size adaptive area opening from a Kinect camera scene: (a) image representing the level of intensity of the scene; (b) image representing the depth of the scene (more a pixel is bright further it would be), (c) and (e) represent the classical area opening, (d) and (f) represent the size adaptive area opening, and $(\mathrm{g})$ and $(\mathrm{h})$ represents the difference between classical area opening and size adaptive area opening.

However sometimes we need a tool that is able to treat both foreground and background of an image in the same way. By this motivation, we arrive at a different morphology which is intrinsically self-dual [14] [16]. More precisely, we want to work with self-dual operators, which means that if $\Psi$ is an operator, we have $\Psi(f)=\complement \Psi(\complement f)$ for every image $f$.

The main ingredient of self-dual morphological operators is a new partial order, as the one depicted in Figure 3(a), such that given two points $s$ and $t$, then $s \preceq t$ implies that:

$$
0 \leq s \leq t \text { or } t \leq s \leq 0
$$

Partial order (15) depends on a constant reference at 0 . More generally, if we consider two spatial functions (i.e., images) $f$ and $g$ and an additional spatially-variant reference $r$, such as the example given in Figure 3(b), we introduce a
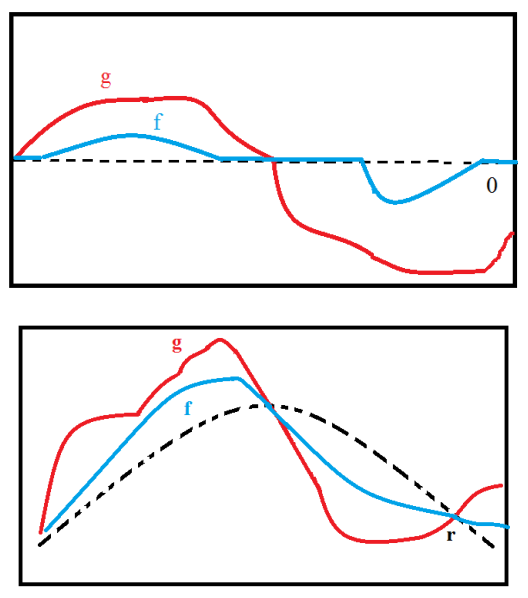

Fig. 3. Top, example of two ordered images $f$ and $g$ such that $f \preceq g$. Bottom example of two ordered images $f$ and $g$ with respect to a reference $r$ such that $f \preceq_{r} g$.

spatially-variant partial order driven by $r$ as:

$$
f \preceq_{r} g \Leftrightarrow \forall x\left\{\begin{array}{l}
g(x) \geq f(x) \geq r(x) \text { if } g(x) \geq r(x) \\
g(x) \leq f(x) \leq r(x) \text { if } g(x)<r(x)
\end{array}\right.
$$

Thanks to Propositions 5.1 and 5.2 of [16], it is possible to define a self-dual opening $\widehat{\gamma_{\lambda, r}}$ on image $f \in \mathcal{F}(E, \mathcal{T})$ with respect to reference image $f \in \mathcal{F}(E, \mathcal{T})$ from an opening $\gamma_{\lambda}$ as

$$
\widehat{\gamma_{\lambda, r}}=\gamma_{\lambda}((f-r) \vee 0)-\gamma_{\lambda}((r-f) \vee 0)+r
$$

which is idempotent, anti-extensive, and increasing according to the partial ordering (16), so it is an algebraic opening. However, this operator do not preserve the connected components if the reference $r$ is not constant. That means that even if $\gamma_{\lambda}$ is a connected filter, the straightforward self-dual counterpart $\widehat{\gamma_{\lambda, r}}$ is not.

That is why we decided to work again on at the connected component level. Obviously there are two cases, all the pixels of the connected component of image $f$ are above the one of reference $r$ so we apply a classical area opening. The second case is similar, it is when all the pixel of the connected component of image $f$ are bellow the one of reference $r$, so also in this case we apply a classical area closing. However there is a third case, where we can apply in an object both closing and opening. In order to solve this ambiguous case, we decided to create a third spatially-variant zone where the identity is applied.

\section{B. Definition}

Let us focus on a formal definition of our self-dual area opening. Like with the previous opening we want to implement the opening on upper level sets. In this case, we have choose to build a trinary upper level set $\mathcal{T}=\{-1,0,1\}$, by calculating the binary upper level set and putting all null pixels to -1 on the trinary upper level set.

Then, let us decompose our trinary image, whose values belong to $\{-1,0,1\}$, in a set of connected objects. The trinary image can be decomposed into three sets of connected objects, 
one corresponding to the objects whose value is $1: \mathcal{C}_{f}^{+}$; one to the objects whose value is $-1: \mathcal{C}_{f}^{-}$; and one to the objects whose value is $0: \mathcal{C}_{f}^{0}$. An example of such image is given in Figure 4, where $\operatorname{card}\left(\mathcal{C}^{+}\right)=6, \operatorname{card}\left(\mathcal{C}^{-}\right)=3, \operatorname{card}\left(\mathcal{C}^{0}\right)=1$. It is now possible to decompose $f$ and $r, f=\mathcal{C}_{f}^{+} \cup \mathcal{C}_{f}^{-} \cup \mathcal{C}_{f}^{0}$ and also for $r=\mathcal{C}_{r}^{+} \cup \mathcal{C}_{r}^{-} \cup \mathcal{C}_{r}^{0}$.

We consider again a Boolean function $v$ that for each connected component $C_{k} \in \mathcal{C}_{f}^{+} \cup \mathcal{C}_{f}^{-}$measures the area and checks if it is greater than $\lambda$ :

$$
v\left(C_{k}\right)=\left\{\begin{array}{l}
1 \text { if area }\left(C_{k}\right) \geq \lambda \\
0 \text { otherwise }
\end{array}\right.
$$
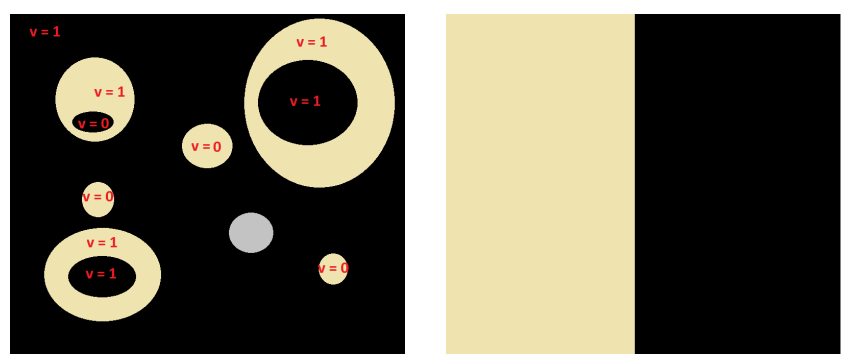

Fig. 4. Example of trinary-valued upper level set of an image $f$ (left) and a reference $r$ (right).

We introduce now an additional Boolean function $u$ that would check if the objects are in the "opening area", or in the "closing area", or on the "identity area". Therefore, $\forall C_{k} \in \mathcal{C}_{f}^{+}$, we have

$$
u\left(C_{k}\right)=\left\{\begin{array}{l}
1 \text { if } C_{k} \subset \mathcal{C}_{r}^{-} \\
0 \text { otherwise }
\end{array}\right.
$$

and $\forall C_{j} \in \mathcal{C}_{f}^{-}$

$$
u\left(C_{j}\right)=\left\{\begin{array}{l}
1 \text { if } C_{j} \subset \mathcal{C}_{r}^{+} \\
0 \text { otherwise }
\end{array}\right.
$$

Thus we can now define the area opening on the trinary image $f$ according to the trinary reference $r$ as:

$$
\begin{array}{r}
\widetilde{\gamma_{\lambda, r}}(f)=\bigcup\left\{C \mid\left(C \subset \mathcal{C}_{f}^{+}\right) \&(u(C)=1) \&(v(C)=1)\right\}+ \\
\bigcup\left\{C \mid\left(C \subset \mathcal{C}_{f}^{+}\right) \&((u(C)=0)\}-\bigcup\left\{C \mid\left(C \subset \mathcal{C}_{f}^{-}\right) \&(u(C)=0)\right\}\right. \\
-\bigcup\left\{C \mid\left(C \subset \mathcal{C}_{f}^{-}\right) \&(u(C)=1) \&(v(C)=1)\right\}
\end{array}
$$

Now, using (5), we can define a self-dual area opening $\widetilde{\gamma_{\lambda, r}}(f)$ on grey-scale image $f$ according to reference $r$ as the sum of the area opening (20) applied in all upper level sets considered as trinary images, i.e.

$$
\widetilde{\gamma_{\lambda, r}}(f)(x)=\sum_{s \in \mathcal{T}} \widetilde{\gamma_{\lambda, r}}\left(X_{s}(f)\right)(x) \text {. }
$$

\section{Proof of the opening}

g) Anti-extensive: Since the original image $f$ is a trinary valued function, we can decompose the study in three cases. First case, $f(x)$ is white so its value is " +1 ", so this pixel is in an object that belong to $\mathcal{C}_{f}^{+}$. From the definition of the previous operator there are two options. First one, the result is white valued so: $\widetilde{\gamma_{\lambda, r}}(f)(x)=1$, or the result is grey valued so $\widetilde{\gamma_{\lambda, r}}(f)(x)=0$. In both cases we have that: $\widetilde{\gamma_{\lambda, r}}(f)(x) \preceq f(x)$. Using exactly the same demonstration, we can assert that if $f(x)=-1$ we also have $\widetilde{\gamma_{\lambda, r}}(f)(x) \preceq f(x)$. If $f(x)=0$ we have $\widetilde{\gamma_{\lambda, r}}(f)(x)=0$. Hence this operator is anti-extensive.

h) Increasing: Now let us consider two trinary images $f$ and $g$, we have the following property:

$$
f \preceq g \Leftrightarrow\left\{\begin{array}{l}
\mathcal{C}_{f}^{+} \subseteq \mathcal{C}_{g}^{+} \\
\mathcal{C}_{f}^{-} \subseteq \mathcal{C}_{g}^{-}
\end{array}\right.
$$

Now let us consider $C$ an object from $\mathcal{C}_{f}^{+}$. There are two possibilities according to the operator. It is possible that $\widetilde{\gamma_{\lambda, r}}(f)(C) \subset \mathcal{C}_{f}^{+}$or $\widetilde{\gamma_{\lambda, r}}(f)(C) \subset \mathcal{C}_{f}^{0}$. If $\widetilde{\gamma_{\lambda, r}}(f)(C) \subset$ $\mathcal{C}_{f}^{+}$then $\widetilde{\gamma_{\lambda, r}}(g)(C) \subset \mathcal{C}_{g}^{+}$because $f \preceq g$. So we have $\mathcal{C}_{\overline{\gamma_{\lambda, r}}(f)}^{+} \subseteq \mathcal{C}_{\overline{\gamma_{\lambda, r}}}^{+}(g)$. Using the same demonstration, we have $\mathcal{C}_{\widetilde{\gamma_{\lambda, r}}(f)}^{-} \subseteq \mathcal{C}_{\widetilde{\gamma_{\lambda, r}}(g)}^{-}$. So we have $\widetilde{\gamma_{\lambda, r}}(f) \preceq \widetilde{\gamma_{\lambda, r}}(g)$. Hence this operator is increasing.

i) Idempotent: Let us consider an object $C \subset \mathcal{C}_{f}^{+}$. From the definition of the operator there are two possibilities, either $\widetilde{\gamma_{\lambda, r}}(f(C)) \subset \mathcal{C}_{\gamma_{\lambda, r}}^{+}(f)$ or $\widetilde{\gamma_{\lambda, r}}(f(C)) \subset \mathcal{C}_{\widetilde{\gamma_{\lambda, r}}(f)}^{0}$.

If $\widetilde{\gamma_{\lambda, r}}(f(C)) \subset \mathcal{C}_{\overline{\gamma_{\lambda, r}}}^{+}(f)$, then easily we have $\widetilde{\gamma_{\lambda, r}}(f(C))=C$. So $u(C)=u\left(\widetilde{\gamma_{\lambda, r}}(f(C))\right)$ and $v(C)=$ $v\left(\widetilde{\gamma_{\lambda, r}}(f(C))\right)$, then we finally have $\widetilde{\gamma_{\lambda, r}}\left(\widetilde{\gamma_{\lambda, r}}(f(C)) \subset\right.$ $\mathcal{C}_{\gamma_{\lambda, r}}^{+}\left(\widetilde{\gamma_{\lambda, r}}(f(C))\right.$, therefore $\widetilde{\gamma_{\lambda, r}}(f(C))=\widetilde{\gamma_{\lambda, r}}\left(\widetilde{\gamma_{\lambda, r}}(f(C))\right.$.

If $\widetilde{\gamma_{\lambda, r}}(f(C)) \subset \mathcal{C}_{\widetilde{\gamma_{\lambda, r}}}^{0}$, then easily we have $\widetilde{\gamma_{\lambda, r}}\left(\widetilde{\gamma_{\lambda, r}}(f(C)) \subset \mathcal{C}_{\widetilde{\gamma_{\lambda, r}}}^{0}\left(\widetilde{\gamma_{\lambda, r}}(f(C))\right.\right.$, and $\widetilde{\gamma_{\lambda, r}}(f(C))=$ $\widetilde{\gamma_{\lambda, r}}\left(\widetilde{\gamma_{\lambda, r}}(f(C))\right.$.

\section{Application to differential image processing}

First to see the result of this opening we tried with simple images and a non flat reference in Figure 5, where we see that this opening is self-dual and does not create discontinuities on connected components. We also try in Figure 6 to apply this opening in video-surveillance applications. We took as images $f$ (a) and $r$ (b) two different frames of a video. One can see the result of the opening in images (c) and (d), and the residues 20)which represent the detailed erased by the opening on images (e) and (f). These residues illustrate the fact that bright and dark objects are similarly affect by the self-dual area opening.

We note that $\widetilde{\gamma}_{\lambda, r}(f) \neq \widetilde{\gamma}_{\lambda, f}(r)$ and consequently, this asymmetry should be taken into account for differential image analysis. In the case of videosequences, it seems natural either to consider $r$ as the frame at time $t-1$ and $f$ the frame at time $t$ or to consider $r$ as a background image of the scene (learned online or fixed) and $f$ each one of the frames. We should point out that the use of area openings for comparison of images was already consider in [3], however in a different framework since here we consider explicitly the computation of the self-dual area opening driven by a reference image and not only shape reconstruction of one image with the other as in [3]. 


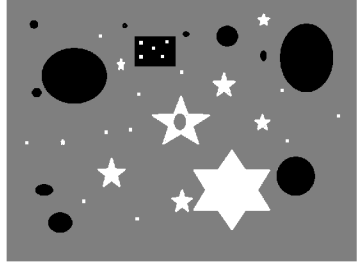

(a) image $f$

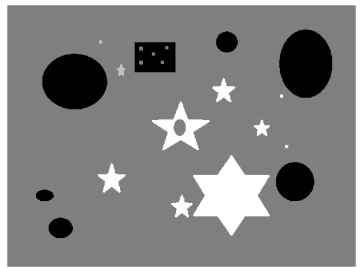

(c) $\widetilde{\gamma}_{500, r}(f)$

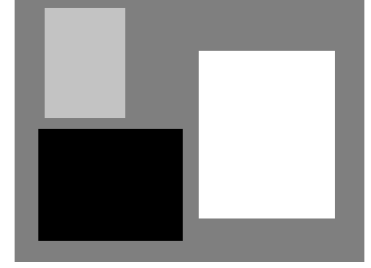

(b) reference $r_{2}$

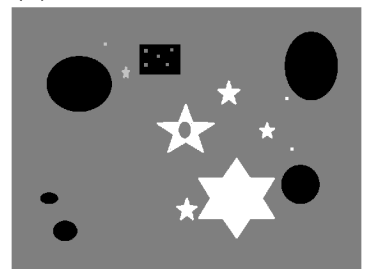

(d) $\widetilde{\gamma}_{5000, r}(f)$

Fig. 5. Examples of spatially-variant self-dual area opening with non-flat reference $\frac{\gamma_{\lambda, r_{2}}}{\gamma_{1}}(f)$

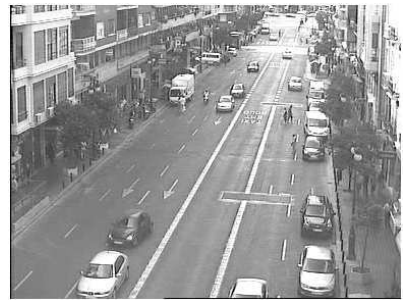

(a) image $f$

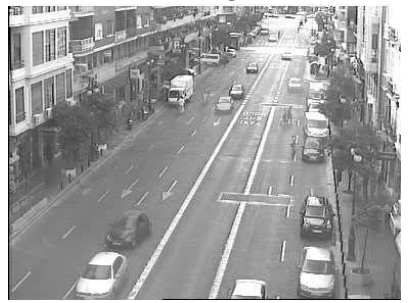

(c) $\widetilde{\gamma}_{500, r}(f)$

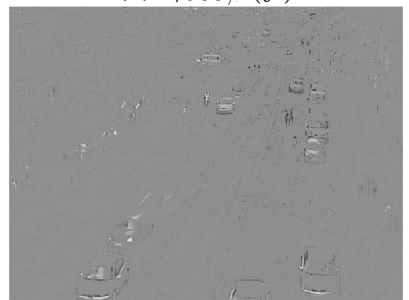

(e) $f-\widetilde{\gamma}_{500, r}(f)$

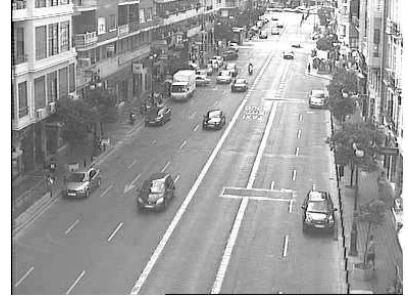

(b) reference $r$



(d) $\widetilde{\gamma}_{5000, r}(f)$

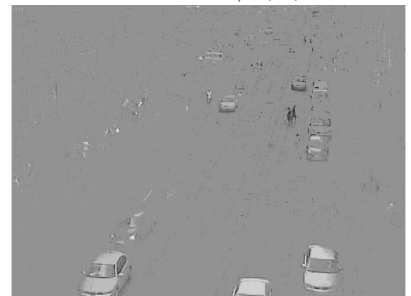

(f) $f-\widetilde{\gamma}_{5000, r}(f)$

Fig. 6. Examples of spatially-variant self-dual area opening with non-flat reference from video-surveillance domain.

\section{COnclusions And PeRspectives}

The purpose of this study was to build two adaptive connected morphological filters that could operate on two images using the information from a image to adapt the effect of the filter on the other image. Both have been theoretically characterized to validate their properties. They can be implemented straightforward using upper-level set decomposition and connected component selection. More efficient algorithms should be considered in ongoing developments.

From an applicative viewpoint, both operators were motivated by practical current problems. The first one is a scaleadaptive 2D image processing according to the scene depth. That allows to filter out objects of same size in 3D scene invariantly to their projection into the $2 \mathrm{D}$ image. The second one involves a self-dual processing of an image according to a background or reference image. The classical framework of application is image sequence processing using a reference scene or the frame $t-1$ as reference to process frame $t$. For instance, it can be used for novelty detection in videosurveillance applications but also in image denoising and regularization of an image according to the reference.

\section{REFERENCES}

[1] Beucher S., "Numerical residues, Image and Vision Computing", Volume 25, Issue 4, April 2007, Pages 405-415.

[2] Angulo J., "Morphological Bilateral Filtering". SIAM Journal on Imaging Sciences, Vol. 6, Issue 3, 1790-1822, 2013.

[3] Monasse P.; Guichard F., "Fast computation of a contrast-invariant image representation," Image Processing, IEEE Transactions on , vol.9, no.5, pp.860-872, May 2000.

[4] Najman L., Couprie M., "Building the Component Tree in Quasi-Linear Time," IEEE Trans. Image Processing, vol. 15, no. 11, pp. 3531-3539, Nov. 2006.

[5] Meyer F., Maragos P., "Nonlinear scale-space representation with morphological levelings." J. Visual Commun. and Image Representation, 11:245-265, 2000 .

[6] Ouzounis G.K., Pesaresi M., Soille P., "Differential Area Profiles: Decomposition Properties and Efficient Computation," Pattern Analysis and Machine Intelligence, IEEE Transactions on , vol.34, no.8, pp.15331548, Aug. 2012.

[7] Salembier P., Serra J., "Flat Zones Filtering, Connected Operators, and Filters by Reconstruction," IEEE Trans. Image Processing, vol. 4, no. 8, pp. 1153-1160, Aug. 1995.

[8] Salembier P., Oliveras A., Garrido L., "Anti-Extensive Connected Operators for Image and Sequence Processing," IEEE Trans. Image Processing, vol. 7, no. 4, pp. 555-570, Apr. 1998.

[9] Serna A., Marcotegui B., "Attribute Controlled Reconstruction and Adaptive Mathematical Morphology," In Mathematical Morphology and Its Applications to Signal and Image Processing (Proc. of ISMM'2013) LNCS Vol. 7883, pp. 207-218, 2013.

[10] Soille P., "Beyond self-duality in morphological image analysis", Image and Vision Computing, Volume 23, Issue 2, 1 February 2005, Pages 249257.

[11] Verdú R., Angulo J., Serra J., "Anisotropic morphological filters with spatially-variant structuring elements based on image-dependent gradient fields," IEEE Trans. on Image Processing, Vol. 20, No. 1, 200-212, 2011.

[12] Maragos P., Schafer R.W., "Morphological filters-Part II: Their relations to median, order-statistic, and stack filters," Acoustics, Speech and Signal Processing, IEEE Transactions on , vol.35, no.8, pp.1170-1184, Aug 1987.

[13] Vincent L., "Morphological area opening and closing for grayscale images," Proc. NATO Shape in Picture Workshop, Driebergen, The Netherlands, Springer-Verlag, pp. 197-208, September 1992.

[14] Heijmans H. J. A. M., Keshet R., "Inf-semilattice approach to self-dual morphology," J. Math. Imaging Vis., 17(1):55-80, July 2002.

[15] Heijmans H. J. A. M., Self-dual morphological operators and filters. J. Math. Imaging Vis., 6(1):1536, Jan. 1996.

[16] Heijmans H. J. A. M., Keshet R., "First steps towards a self-dual morphology," Proceedings of the IEEE International Conference on Image Processing, Vancouver, Canada, Sep 2000.

[17] Serra J., "Image Analysis and Mathematical Morphology. Academic Press,” Inc., Orlando, FL, USA, 1983. 